

\title{
Um estudo das habilidades sociais em idosos
}

\author{
A study of the social skills of the elderly
}

Rachel Shimba Carneiro ${ }^{[a]}$

\footnotetext{
${ }^{[a]}$ Doutora em Psicologia Social pela Universidade do Estado do Rio de Janeiro. É professora do mestrado em psicologia da Universidade Católica de Petrópolis e docente do curso de psicologia da UNISUAM, Rio de Janeior, RJ Brasil, e-mail: rachelshimba@ yahoo.com.br
}

Recebido: 03/03/2013 Received: 03/03/2013

\section{Resumo}

Pesquisas recentes têm mostrado que não se deve aceitar apenas a longevidade do ser humano como a principal conquista da humanidade contemporânea, mas buscar ter garantia de uma vida com qualidade e participação ativa. Assim, uma questão que se torna importante é como obter, manter ou garantir saúde na velhice. Um levantamento bibliográfico do tema em questão revelou as habilidades sociais como um dos fatores importantes para a saúde do idoso. Por outro lado, a deficiência em habilidades de interação pode provocar consequências sérias como transtornos psicológicos, desajustamento e marginalidade. 0 presente estudo teve como objetivo investigar as habilidades sociais de 30 idosos de uma universidade da terceira idade e 15 idosos que vivem em asilos. Os conhecimentos gerados a partir deste estudo fornecem contribuições importantes sobre as habilidades sociais na terceira idade. [\#]

Palavras-chave: Relações interpessoais. Idoso. Saúde do idoso.

\section{Abstract}

Recent research has shown that one should not accept only the longevity of human beings as the main achievement of contemporary humanity, but seek to have a lifetime guarantee of quality and active participation. Thus, an issue that becomes important is how to obtain, maintain or ensure health in old age. A bibliographic survey of the subject revealed social skills as one of the important health factors for the elderly. On the other hand, deficiency in interaction skills can cause serious consequences such as psychological disorders, maladjustment and delinquency. The present study aimed to investigate the social skills of 30 elderly students from a university of the third age and 15 who in nursing homes. The knowledge generated from this study provides important contributions on social skills in old age.

Keywords: Interpersonal relations. Aged. Health of the Elderly.

Psicol. Argum., Curitiba, v. 32, n. 76, 23-31 


\section{Introdução}

O crescimento da população de idosos é um acontecimento mundial e ocorre em um nível sem precedentes (Pereira, Curioni \& Veras, 2003). Em 1950 , havia cerca de 204 milhões de idosos no mundo, em 1998, menos de cinco décadas depois, esse contingente alcançava 579 milhões de pessoas; um crescimento de quase oito milhões de idosos por ano (IBGE, 2002). 0 filósofo alemão Schirrmacher (2004) chegou a afirmar, em uma entrevista consentida nas páginas amarelas da revista Veja, que a humanidade está envelhecendo numa rapidez nunca vista antes. Beauvoir (1990) já chamava atenção para esta questão, na década de 1970 , ao escrever em seu livro A Velhice, o seguinte: "de todos os fenômenos contemporâneos, o menos contestável, o mais fácil de ser previsto com grande antecedência é o envelhecimento das populações" (p. 247).

Para Veras (2003), o crescimento demográfico do grupo etário dos idosos está longe de se estabilizar. Segundo este autor, ainda haverá algumas décadas de ampliação da proporção deste contingente populacional. As projeções indicam que em 2050, a população idosa será de 1.900 milhões de pessoas (IBGE, 2002). Gorzoni (1993) relata que esse aumento da população mundial de idosos é consequência, basicamente, do decréscimo das taxas de natalidade e do desenvolvimento científico que aumentou a expectativa de vida (Merlin, Baptista \& Baptista, 2004), provocando a queda das taxas de mortalidade. Assim, pode-se pontuar que o envelhecimento populacional (Camarano, 2002), no mundo, é um processo decorrente das mudanças nas taxas de mortalidade e natalidade, que segundo Merlin, Baptista \& Baptista (2004) ocorreram a partir da década de 1960. De acordo com Zimerman (2000), a Islândia, a Itália, o Japão e a Suécia estão no topo da lista dos países com população de maior longevidade.

É importante salientar que no Brasil a situação não é diferente e pouco a pouco o mito de um país de jovens vai sendo derrubado (Zimerman, 2000). As estatísticas nacionais recentes indicam que o contingente de idosos, no Brasil, tem crescido de forma considerável (Fonseca \& Gonçalves, 2003). Como afirma Veras (2003), o Brasil é um país que envelhece a passos largos. Em 2002, esse país já possuía em torno de 15 milhões de idosos (Camarano, 2002). Uma projeção para o ano de 2020, segundo mostra estudos de Costa (2001), quando a expectativa de vida estiver alcançando 75,5 anos, a população brasileira será formada por cerca de $23,5 \%$ de jovens e $7,7 \%$ de idosos, ou seja, será composta por 16,2 milhões de idosos. De acordo com Veras (2003), nunca é demais lembrar que o brasileiro tinha a expectativa de vida em 1900, de 33 anos, e em 2000 de 68 anos, ou seja, o tempo de vida de um brasileiro foi dobrado em apenas cem anos. Do ponto de vista deste autor, essas alterações na dinâmica da população são claras, inexoráveis e irreversíveis.

Com base nos dados demográficos anteriormente referidos é possível perceber um grande aumento da proporção de idosos no Brasil com o passar dos anos. Pereira, Curioni e Veras (2003) apontam que esse crescimento é mais significativo no estado do Rio de Janeiro. Neste estado e, em particular a sua capital, apresenta a maior incidência percentual de idosos no país (Frutuoso, 1999). Todos esses dados suscitam uma questão relevante: o Brasil está envelhecendo e é preciso saber como lidar com essa nova realidade.

O reconhecimento do rápido aumento do número de pessoas idosas, nas últimas décadas, ocasionou a explosão de trabalhos científicos sobre o envelhecimento, tanto nas ciências naturais quanto na área das humanidades (Baltes, 1995). Esses estudos têm mostrado que o envelhecer não precisa necessariamente ser acompanhado de perdas ou afastamento social (Capitanini, 2000).

Diante de tais considerações, Xavier et al. (2000) realizaram um estudo sobre as condições psicológicas, sociais e de saúde geral de um grupo representativo de idosos com mais de 80 anos residentes da cidade de Veranópolis/RS. Os resultados desse estudo mostraram evidências contrárias à impressão de que a velhice seja necessariamente indissociável de perda de funcionalidade, saúde e satisfação de viver. Deste modo, estes autores constataram que idades acima de 80 anos não são tempos de pouca saúde e insatisfação com a vida.

Vale ressaltar que a neurociência já não trabalha mais com a hipótese de que a perda cognitiva com o envelhecimento é inevitável (Herculano-Houzel, 2007). Como propõe tal autor, o número de pessoas na terceira idade cujas habilidades mentais são semelhantes às dos moços é grande o suficiente para que elas não possam mais ser consideradas uma exceção. Ainda dentro desta discussão, Goleman (2006) sustenta que: 
A neurogênese, produção diária de novos neurônios pelo cérebro, continua na velhice, embora num ritmo mais lento do que nas décadas anteriores. E essa diminuição do ritmo não precisa ser inevitável, sugerem alguns neurocientistas, mas apenas um efeito colateral da monotonia. Acrescentar complexidade ao ambiente social da pessoa estimula o aprendizado de coisas novas, acelerando o ritmo de produção de novas células pelo cérebro. Por esse motivo, alguns neurocientistas estão trabalhando com arquitetos para projetar lares para os idosos nos quais os habitantes tenham de interagir com os outros durante a execução de sua rotina diária (p. 276-277).

Dentro deste contexto, é possível pontuar que "o envelhecimento é inexorável, resultado da passagem do tempo. Mas ele não é sinônimo de adoecimento nem de perda da qualidade de vida ou do bem-estar" (Herculano-Houzel, 2007, p. 136). Assim, torna-se premente a necessidade de pesquisas, tanto epidemiológicas como de intervenções, que possam identificar como obter, manter e garantir saúde na velhice.

0 argumento em favor da ideia de que as relações sociais podem, de várias formas, promover melhores condições de saúde tem sido salientada em vários estudos (Ramos, 2002). Capitanini (2000) e Ramos (2007), por exemplo, ressaltam a importância dos relacionamentos sociais para o bem-estar físico e mental e para qualidade de vida na velhice. Por outro lado, a ausência de convívio social causa severos efeitos negativos na capacidade cognitiva geral (Katz \& Rubin, 2000), além de depressão e estresse (Freire \& Sommerhalder, 2000). A pobreza de relações sociais como um fator de risco à saúde tem sido considerada tão danosa quanto o fumo, a pressão arterial elevada, a obesidade e a ausência de atividade física (Goleman, 2006; Herculano-Houzel, 2007). Tal assertiva indica que a deterioração da saúde pode ser causada não somente por um desgaste natural do organismo, sedentarismo ou uso de tabaco, mas também, pela redução da quantidade ou qualidade das relações sociais (Ramos, 2002). É interessante notar que mesmo entre macacos, que não fumam, não bebem ou usam drogas, não são sedentários e nem entopem as suas artérias com frituras, vivem mais e melhor aqueles que são bem relacionados e têm o apoio de parceiros estáveis (Herculano-Houzel, 2007).

De um modo geral, os estudos têm mostrado que as pessoas que possuem maior contato social vivem mais e com melhor saúde do que aquelas com menor contato social. Chor, Griep, Lopes e Faerstein (2003) citam algumas pesquisas que constataram que os indivíduos isolados socialmente apresentaram risco de morrer de duas a cinco vezes maior, quando comparados àqueles que mantêm vínculos fortes com amigos, parentes ou grupos. Como afirma Soares (2004) em uma reportagem na revista Veja, intitulada "Viver mais e Melhor", a participação social parece ser um dos fatores importantes na diminuição da mortalidade dos mais velhos. Este aspecto também já vinha sendo destacado por Silberman et al. (1995), no qual apontam que aqueles idosos participantes de algum tipo de atividade social e que visitam ou conversam com amigos ou familiares reduzem o seu risco de mortalidade para quase metade. Diante de todas essas considerações, pode-se perceber que as relações sociais são fontes protetoras e mantenedoras de saúde (Matsukura, Marturano \& Oishi, 2002) e de grande importância em todos os momentos da vida (Resende, Bones, Souza \& Guimarães, 2006).

Seguindo os estudos apresentados anteriormente, Chelala (1992, citado por Oliveira, Pasian \& Jacquemin, 2001) alerta para o fato de que a qualidade dos contatos sociais é mais importante do que a quantidade. Tal fato identifica as habilidades sociais como um dos fatores importantes para a saúde do idoso. É importante notar que ainda não existe um consenso quanto a uma definição de habilidades sociais.

Não é nada fácil, na vasta literatura sobre tais habilidades, encontrar uma definição única (Carmona \& Melo, 2000). Porém, é necessário que se reforce a diferença entre os termos habilidades sociais, competência social e desempenho social, para o uso cuidadoso desses conceitos (Del Prette \& Del Prette, 2001b; 2005).

Em uma revisão de estudos feita por Bandeira e Quaglia (2005) foi verificada que as habilidades sociais são unidades comportamentais que fazem parte do desempenho do indivíduo diante das demandas das situações interpessoais e que são necessárias à competência social. Para Del Prette e Del Prette (2001b), o termo habilidades sociais refere-se à existência de diferentes classes de comportamentos sociais no repertório do indivíduo para lidar de maneira adequada com as demandas das situações interpessoais. Dessa forma, para estes autores, as principais classes das habilidades sociais são:

Psicol. Argum., Curitiba, v. 32, n. 76, 23-31 
habilidades sociais de comunicação; habilidades sociais de civilidade; habilidades sociais assertivas, de direito e cidadania; habilidades sociais empáticas; habilidades sociais de trabalho e habilidades sociais de expressão de sentimento positivo.

Já a competência social remete ao conjunto de comportamentos demonstrados pelo indivíduo, num contexto interpessoal, em que expressa sentimentos, atitudes, desejos, opiniões, direitos, de forma coerente com a situação, respeitando os comportamentos dos outros e que de forma geral solucione os problemas imediatos, reduzindo assim a possibilidade de conflitos no futuro (Caballo, 1987). Dessa forma, Del Prette e Del Prette (2005) afirmam que a competência social deve ser compreendida "sob uma perspectiva avaliativa a respeito da proficiência do desempenho" (p. 8). Por fim, o desempenho social "refere-se à emissão de um comportamento ou sequência de comportamentos em uma situação qualquer" (Del Prette \& Del Prette, 2001b, p. 31).

Do ponto de vista de Vila (2005), é importante destacar que como a variedade de contextos sociais requer a emissão de desempenhos sociais bastante diferenciados, para que o desempenho social seja considerado competente, é de extrema importância que se faça uma leitura das "dicas sociais" implícitas nos diferentes contextos sociais. Tal colocação pressupõe que o indivíduo socialmente competente deve ter um conhecimento não somente da resposta adequada, mas também de como e quando essa conduta pode ser emitida (Carmona \& Melo, 2000).

Dentro deste contexto, pode-se afirmar que o comportamento socialmente competente não compreende apenas desempenho aberto (verbais e não verbais), mas também componentes cognitivos de atenção, percepção e processamento de informação. Ou seja, a competência social possibilita ao indivíduo discriminar como deve se comportar nas diferentes situações sociais (Vila, 2005). Além disso, Falcone e Ramos (2005) consideram que o conceito de comportamento socialmente competente inclui a capacidade do indivíduo para obter satisfação pessoal (assertividade) e, ao mesmo tempo, a motivação genuína para compreender e atender às necessidades da outra pessoa (empatia).

Diante das considerações anteriores nota-se que as interações sociais bem sucedidas incluem a manifestação da habilidade empática e da habilidade assertiva. A empatia é entendida como a capacidade de compreender e de expressar compreensão acurada sobre a perspectiva e sentimentos de outra pessoa, além de experimentar compaixão e interesse pelo bem-estar desta (Barrett-Lennard, 1993). Do ponto de vista de Falcone (1998), agir de forma empática implica estar disponível para ver as coisas de acordo com o ponto de referência da outra pessoa e demonstrar um interesse genuíno pelo bem-estar dela. Deste modo, os indivíduos empáticos tornam as relações mais agradáveis, reduzindo o conflito e o rompimento (Falcone, 1998). Por outro lado, indivíduos não empáticos parecem carecer de inteligência social e podem se tornar prejudicados no trabalho, na escola, na vida conjugal, nas amizades e nas relações familiares, além de correrem o risco de viver à margem da sociedade (Goleman, 1995).

Já a assertividade é definida, por Lange e Jakubowski (1976), como a "capacidade de defender os próprios direitos e de expressar pensamentos, sentimentos e crenças de forma honesta, direta e apropriada, sem violar os direitos da outra pessoa" (p. 7), possibilitando bem-estar emocional e aumentando a probabilidade da manutenção de relacionamentos interpessoais saudáveis e duradouros (Vila, Gongora \& Silveira, 2003).

Seguindo os estudos apresentados anteriormente, Falcone (1998) declara que expressar-se de maneira empática antes de usar a assertividade direta pode minimizar qualquer avaliação negativa potencial da assertividade. Com base nesta proposição, esta autora cita alguns estudos ao mostrar que, em determinados contextos sociais, especialmente quando há conflito, torna-se necessário controlar as próprias emoções e fazer um esforço para compreender e validar os sentimentos e a perspectiva da outra pessoa, antes da manifestação dos próprios sentimentos e perspectiva. Tais argumentos sugerem que o sucesso nas relações interpessoais depende da integração das habilidades: empática e assertiva.

De uma maneira geral, pode-se dizer que os déficits e comprometimentos de habilidades sociais podem caracterizar relações sociais restritas e conflitivas que interferem, de maneira negativa, na qualidade de vida e na saúde psicológica do indivíduo (Del Prette \& Del Prette, 2005; Magalhães \& Murta, 2003). Apesar de reconhecer a importância do repertório social do idoso, ainda são ínfimos estudos de avaliação nessa área. 
Em um estudo realizado por Deps (1993) foi investigado a ocupação do tempo livre de 63 idosos residentes em instituições. Com base nas observações de campo, tal autora descreveu as atividades desempenhadas pelos idosos institucionalizados e, subsequentemente, apontou aspectos que podem estar dificultando a interação entre esses idosos. Para a coleta de dados, foram utilizados uma entrevista semiestruturada, o teste sociométrico (Alves, 1964) e a observação direta. Os resultados do teste sociométrico refletiram o isolamento, em que esses idosos viviam, ou a pouca comunicação entre eles. Tal dado foi confirmado pela observação direta da autora, verificando uma apatia visível nesses idosos, os quais ficavam sentados um ao lado do outro, mas sem conversarem.

Em seu estudo, Deps (1993) destacou nove motivos para os idosos conversarem tão pouco entre si: (1) convívio dificultado por desentendimento, agressividade, intrigas, senilidade, indiferença e egoísmo; (2) doença (dores, falta de ar, deficiência auditiva); (3) falta de assunto por não saírem ou não lerem; (4) assunto desinteressante; (4) falta de memória; (5) temperamento (não gosta de conversar); (6) pouco conhecimento entre eles; (7) diferença de idade; (8) necessidade de silêncio e (9) distanciamento físico. Além disso, é importante constatar que Deps (1993) verificou: (1) pouco ou nenhum suporte emocional das pessoas que trabalhavam nas instituições ou dos próprios idosos residentes dos asilos para superarem as dificuldades pessoais dos companheiros; (2) restrições impostas pelo ambiente institucional; (3) oferecimento de atividades limitadas, sem planejamento; (4) oportunidades ocupacionais pouco vinculadas aos reais interesses dos idosos; (5) cuidadores leigos influenciados por concepções negativas do processo de envelhecimento, oriundas do contexto social e (6) ambiente físico pouco propício à prática de atividades. Diante de tais considerações, Deps (1993) chama atenção para o fato de que os asilos investigados basicamente só levam em conta as necessidades biológicas dos indivíduos, esquecendo as necessidades de assistência sociocultural.

Os estudos e argumentos apresentados até agora suscitam a seguinte questão: será que os idosos que frequentam ambientes sociais ricos em relações interpessoais apresentam um maior repertório de habilidades sociais do que os idosos inseridos em ambientes que não possuem essa "riqueza" de relações sociais? Levando em consideração que a privação sociocultural, em casos de institucionalização ou isolamento do indivíduo, pode estar relacionada com dificuldades de interação social, o presente estudo pretende verificar se os idosos que estão inseridos em uma Universidade da Terceira Idade tendem a ser mais socialmente competentes do que idosos saudáveis moradores de asilo.

\section{Método}

\section{Participantes}

A amostra consistiu de 45 idosos (com idades variando de 61 a 95 anos), distribuídos da seguinte forma: 30 idosos que frequentam ambientes de ensino, como a Universidade da Terceira Idade, e 15 idosos que vivem em asilos.

\section{Instrumentos e Material}

Cada participante recebeu o Termo de Consentimento Livre e Esclarecido (TCLE) contendo informações sobre os objetivos da pesquisa e o compromisso com o sigilo a respeito da identidade dos participantes.

Uma Ficha de Entrevista foi utilizada para a obtenção de dados pessoais referentes à amostra estudada (idade, sexo, localização geográfica, estado civil, escolaridade, tempo de ingresso na Universidade ou tempo de residência no asilo).

Utilizado para avaliar as habilidades sociais dos participantes, o Inventário de Habilidades Sociais (IHS-Del-Prette, de Del Prette \& Del Prette, 2001a) é composto de 38 itens que se agrupam em cinco fatores: (1) Enfrentamento e autoafirmação; (2) Autoafirmação na expressão de sentimentos positivos; (3) Conversação e desenvoltura social; (4) Autoexposição a desconhecidos e situações novas; (5) Autocontrole da agressividade. 0 fator 1 (itens $1,5,7,11,12,14,15,16,20,21$ e 29 ) avalia principalmente o conceito de assertividade, reunindo itens que retratam situações interpessoais em que a demanda de reação ao interlocutor se caracteriza principalmente pela afirmação e defesa de direitos e de autoestima, com risco potencial de reação indesejável (possibilidade de rejeição, de réplica ou de oposição) por parte do interlocutor. Já o fator

Psicol. Argum., Curitiba, v. 32, n. 76, 23-31 
2 (itens $3,6,8,10,28,30$ e 35 ) retrata demandas interpessoais de expressão de afeto positivo e de afirmação da autoestima, com risco mínimo de reação indesejável. Apresenta o fator 3 (itens 13, $17,19,22,24,36$ e 37) situações sociais neutras de aproximação com risco mínimo de reação indesejável, demandando, principalmente, "traquejo social" na conversação, o que supõe conhecimento das normas de relacionamento cotidiano. As situações que envolvem a abordagem de pessoas desconhecidas são medidas pelo fator 4 (itens 9, 14, 23 e 26). Por fim, o fator 5 (itens 18, 31 e 38) reúne itens que supõem reação às estimulações aversivas do interlocutor, demandando controle da raiva e da agressividade. Parte dos itens do IHS-Del-Prette é redigida de modo que uma frequência mais elevada indica déficit na habilidade requerida naquela situação (nesses itens a pontuação é invertida para a obtenção do escore). 0 escore total permite uma primeira avaliação dos recursos e déficits das habilidades sociais do respondente, e os escores fatoriais, por sua vez, apresentam as áreas específicas, nas quais estas habilidades ou faltas se apresentam. Conforme consta no Manual, propriedades psicométricas do IHS-Del-Prette apresentam índices satisfatórios de validade, fidedignidade e consistência interna.

É importante considerar que foram realizadas algumas alterações no IHS-Del-Prette para a sua utilização com indivíduos na terceira idade. Os itens $2,5,8,9,10,11,13,14,15,17,18,22,29,34$ e 38 foram modificados sem que houvesse alteração no sentido de suas afirmativas.

\section{Procedimento}

Após aprovação do estudo pela Comissão de Ética em Pesquisa, a pesquisadora entrou em contato com a diretora de um asilo, para explicar os objetivos da pesquisa e solicitar permissão para a realização do estudo. Em seguida, os idosos que voluntariamente concordaram em participar receberam o Termo de Consentimento Livre e Esclarecido. Após a assinatura deste documento, foi entregue a ficha de entrevista, seguida pela aplicação do IHS-Del-Prette que foi realizada individualmente e em um único encontro, além disso, não houve tempo pré-determinado para o preenchimento do Inventário.

\section{Tratamento dos dados}

Inicialmente, de acordo com a instrução normativa do IHS-Del-Prette (Del Prette \& Del Prette, 2001a), foi calculado o escore total de cada respondente, bem como os escores fatoriais de cada participante e os valores médios por grupo em cada um dos fatores. Para a comparação entre o grupo do asilo e o grupo de idosos que frequentam a Universidade da Terceira Idade, foi usada a análise da variância simples (one-way ANOVA) com post-hoc (LSD), com o tipo de grupo entrando como variável independente e os cinco fatores do IHS-Del-Prette como variáveis dependentes. As distribuições das pontuações das variáveis utilizadas foram avaliadas por meio do teste de Kolmogorov-Smirnov para uma amostra, a fim de verificar se estas eram significativamente diferentes das demais. 0 nível de significância adotado para este teste estatístico, bem como para os demais, foi de $5 \%$. Como não foram encontrados desvios significativos da normalidade, foram adotados testes estatísticos paramétricos para as comparações de médias.

\section{Resultados}

Ao comparar o grupo da Universidade da Terceira Idade com o grupo do asilo, foram encontradas diferenças significativas para os fatores: (1) enfrentamento e autoafirmação com risco ( $p=0,001),(2)$ autoafirmação na expressão de sentimentos positivos $(p=0,001),(3)$ conversação e desenvoltura social $(p=0,000)$, e para o fator (4) autoexposição a desconhecidos e situações novas ( $\mathrm{p}=0,001)$. Os resultados obtidos no fator autocontrole da agressividade $(p=0,890)$ não demonstraram diferenças significativas entre os dois grupos pesquisados.

A partir dos resultados anteriores apresentados, pôde-se constatar que os idosos do grupo do asilo apresentaram uma maior deficiência nas situações do Inventário de Habilidades Sociais que avaliavam a habilidade assertiva do que os idosos do grupo da Universidade da Terceira Idade. Tais dados sustentam a afirmação de Gurfein e Stutman (1996), ao apontar que o treinamento de habilidades sociais com o idoso institucionalizado é utilizado para ajudá-lo a desenvolver um comportamento assertivo. É importante salientar que, nos resultados do estudo desenvolvido por Donohue, Acierno, Hasselt 
e Hersen (1995), foi encontrada uma correlação positiva significativa entre a assertividade e a habilidade em obter apoio social do ambiente quando necessário.

Os participantes do grupo do asilo também apresentaram um menor repertório de habilidades sociais, quando comparados com os idosos do grupo da Universidade da Terceira Idade em situações que demandam autoafirmação na expressão de sentimento positivo. Além disso, o grupo do asilo apresentou um menor repertório de habilidades sociais para lidar com situações que envolvem conversação e desenvoltura social e situações de autoexposição a desconhecidos (e.g., de manter e encerrar conversação em contato face a face, encerrar conversa ao telefone, abordar pessoas que ocupam posição de autoridade, pedir favor a colegas e desconhecidos, recusar pedidos abusivos, fazer apresentações ou palestras a um público desconhecido e fazer perguntas a pessoas desconhecidas). Uma possível explicação para esses resultados refere-se ao isolamento em que os idosos residentes em instituições vivem, ou a pouca comunicação entre eles (Deps, 1993).

Cabe a ressalva de que os dois grupos não diferenciaram muito entre si ao reagir em situações de lidar com críticas de familiares e com chacotas ou brincadeiras ofensivas. Os dados apresentados até o momento mostram que os idosos do asilo têm um maior comprometimento nas habilidades sociais do que os estudantes da Universidade da Terceira Idade. De acordo com os estudos de Del Prette e Del Prette (1999), os déficits e comprometimentos de habilidades sociais podem caracterizar relações sociais restritas e conflitivas, comprometendo a obtenção do apoio social e, consequentemente, a qualidade de vida do idoso.

\section{Discussão}

Os dados destacados até o presente momento parecem indicar que os idosos do grupo da Universidade da Terceira Idade possuem uma maior capacidade para se comunicar socialmente do que os idosos do asilo. Esses achados estão em consonância com os estudos desenvolvidos por Deps (1993) e com as afirmações de Neri e Sommerhalder (2001). Os estudos daquela autora mostram que as instituições basicamente só levam em conta as necessidades biológicas dos indivíduos, esquecendo-se das necessidades de assistência sociocultural, e estas autoras assinalam que as redes sociais formadas por familiares e amigos oferecem apoio social na forma de amor, afeição, preocupação e assistência, abalando significativamente os efeitos do estresse e da depressão nos indivíduos mais velhos.

Algumas propostas sugeridas para futuras pesquisas são: o aumento do número de sujeitos da amostra e a avaliação do impacto das habilidades sociais na redução da depressão e, consequentemente, na qualidade de vida do idoso. Além disto, seria interessante que outros trabalhos investigassem a relação entre o contexto social e o repertório de habilidades sociais.

\section{Considerações finais}

O presente estudo procurou trazer algumas contribuições importantes sobre as habilidades sociais na terceira idade, levando-se em conta que é escasso o material disponível sobre o tema. Aprofundar o conhecimento sobre este tema parece relevante, principalmente no contexto atual de mudança do perfil populacional e tecnológico, que amplia a expectativa de vida das pessoas.

\section{Referências}

Alves, D. J. (1964). O teste sociométrico. Rio de Janeiro: Fundação Getúlio Vargas.

Baltes, P. B. (1995). Prefácio. In A. L. Neri, Psicologia do envelhecimento (pp. 9-12). Campinas: Papirus.

Bandeira, M., \& Quaglia, M. A. C. (2005). Habilidades sociais de estudantes universitários: Identificação de situações sociais significativas. Interação em Psicologia, 9(1), 45-55.

Barrett-Lennard, G. T. (1993). The phases and focus of empathy. The British Psychological Society, 66, 3-14.

Beauvoir, S. (1990). A velhice. Rio de Janeiro: Nova Fronteira.

Caballo, V. E. (1987). Teoría, evaluación y entrenamiento de las habilidades sociales. Valencia: Promolíbro.

Psicol. Argum., Curitiba, v. 32, n. 76, 23-31 
Camarano, A. A. (2002). Envelhecimento da população brasileira: Uma contribuição demográfica. In A. L. Neri, E. V. Freitas, F. A. X. Cançado, L. Py, M. L. Gorzoni, S. M. Rocha (Orgs.), Tratado de geriatria e gerontologia (pp. 58-71). Rio de Janeiro: Guanabara Koogan.

Capitanini, M. E. S. (2000). Solidão na velhice: Realidade ou mito? In A. L. Neri \& S. A. Freire (Orgs.), E por falar em boa velhice (pp. 69-89). Campinas: Papirus.

Carmona, C. G. H., \& Melo, N. A. (2000). Comunicación interpersonal: Programa de entrenamiento en habilidades sociales. Santiago: Ediciones Universidad Católica de Chile.

Chor, D., Griep, R. H., Lopes, C. S., \& Faerstein, E. (2003). Social support: Scale test-retest reliability in the pro-health study. Cadernos de Saúde Pública, 19(2), 625-634.

Costa, G. A. (2001). Tempo de ser: Atividade física, qualidade de vida, envelhecimento e a trama das interações sociais interferindo na relação de gênero. Revista da Sobama, 6(1), 9-18.

Del Prette, Z. A. P., \& Del Prette, A. (1999). Psicologia das habilidades sociais: Terapia e educação. Petrópolis: Vozes.

Del Prette, Z. A. P., \& Del Prette, A. (2001a). Inventário de Habilidades Sociais: Manual de aplicação, apuração e interpretação. São Paulo: Casa do Psicólogo.

Del Prette, Z. A. P., \& Del Prette, A. (2001b). Habilidades sociais: Biologia evolucionária e cultura. In H. J. Guilhardi (Org.), Sobre comportamento e cognição: Expondo a variabilidade (pp. 65-75). Santo André: ESETec Editores Associados.

Del Prette, Z. A. P., \& Del Prette, A. (2005). Perguntas (im) pertinentes sobre a área do treinamento das habilidades sociais. In H. J. Guilhardi \& N. C. Aguirre (Orgs.), Sobre comportamento e cognição: Expondo a variabilidade (pp. 5-13). Santo André: ESETec Editores Associados.

Deps, V. L. (1993). A ocupação do tempo livre sob a ótica de idosos residentes em instituições: Análise de uma experiência. In A. L. Neri (Org.), Qualidade de vida e idade madura (pp. 191-211). Campinas: Papirus.
Donohue, B., Acierno, R., Hasselt, V. B. V., \& Hersen, M. (1995). Social skills training in a depressed, visually impaired older adult. J. Behav. Ther. \& Exp. Psychiat, 26(1), 65-75.

Falcone, E. M. O. (1998). A avaliação de um programa de treinamento da empatia. Tese de Doutorado, Universidade de São Paulo, São Paulo.

Falcone, E. M. O., \& Ramos, D. M. (2005). A atribuição como componente cognitivo das habilidades sociais e seu impacto na satisfação conjugal. In H. J. Guilhardi \& N. C. Aguirre (Orgs.), Sobre comportamento e cognição: Expondo a variabilidade (pp. 182-191). Santo André: ESETec Editores Associados.

Fonseca, M. M. da, \& Gonçalves, H. S. (2003). Violência contra o idoso: Suportes legais para a intervenção. Interação em Psicologia, 7(2), 121-128.

Freire, S. A., \& Sommerhalder, C. (2000). Envelhecer nos Tempos Modernos. In A. L. Neri \& S. A. Freire (Orgs.), E por falar em boa velhice (pp. 125-135). Campinas: Papirus.

Frutuoso, D. (1999). A terceira idade na universidade. Rio de Janeiro: Ágora da Ilha.

Goleman, D. (1995). Inteligência emocional. (M. Santarrita Trad.).Rio de Janeiro: Objetiva.

Goleman, D. (2006). Inteligência social: O poder das relações humanas. (A. B. Rodrigues Trad.). Rio de Janeiro: Elsevier.

Gorzoni, M. L. (1993). Aspectos de farmacologia clínica em pacientes idosos. Gerontologia, 1(1), 9-12.

Gurfein, H. N., \& Stutman, G. F. (1996). Psicoterapia de grupo com idosos. In H. I. Kaplan \& B. J. Sadock (Orgs.), Compêndio de Psicoterapia de grupo (pp. 486495). Porto Alegre: Artes Médicas.

Herculano-Houzel, S. (2007). Fique de bem com seu cérebro: Guia prático para o bem-estar em 15 passos. Rio de Janeiro: Sextante.

Instituto Brasileiro de Geografia e Estatística [IBGE]. (2002). Perfil dos idosos responsáveis pelos domicílios 
no Brasil 2000. Rio de Janeiro: IBGE, Departamento de População e Indicadores Sociais.

Katz, L., \& Rubin, M. (2000). Mantenha o seu cérebro vivo. São Paulo: Sextante.

Lange, A., \& Jakubowski, P. (1976). Responsible assertive behavior. Illionis: Ed. Research Press.

Magalhães, P. P., \& Murta, S. G. (2003). Treinamento de habilidades sociais em estudantes de psicologia: Um estudo pré-experimental. Temas em Psicologia da SBP, 11(1), 28-37.

Matsukura, T. S., Marturano, E. M., \& Oishi, J. O. (2002). O questionário de suporte social (SSQ): Estudos da adaptação para o português. Revista LatinoAmericana de Enfermagem, 10(5), 675-681.

Merlin, M. S., Baptista, A. S. D., \& Baptista, M. N. (2004). Depressão e suicídio na terceira idade. In M. N. Baptista, Suicídio e depressão: Atualizações (pp. 195216). Rio de Janeiro: Guanabara Koogan.

Neri, A. L., \& Sommerhalder, C. (2001). As várias faces do cuidado e do bem-estar do cuidador. In A. L. Neri (Org.), Cuidar de idosos no contexto da família: Questões psicológicas e sociais (pp. 9-62). Campinas: Alínea.

Oliveira, E. A. de, Pasian, S. R., \& Jacquemin, A. (2001). A vivência afetiva em idosos. Psicologia Ciência $e$ Profissão, 21(1), 68-83.

Pereira, R. S., Curioni, C. C., \& Veras, R. (2003). Perfil demográfico da população idosa no Brasil e no Rio de Janeiro em 2002. Textos sobre Envelhecimento, 6(1), 43-59.

Ramos, M. P. (2002). Apoio social e saúde entre idosos. Sociologias, 4(7), 156-175.

Ramos, M. (2007). Os sintomas depressivos e as relações sociais na terceira idade. Revista do Departamento de Psicologia, UFF, 19(2), 397-410.
Resende, M. C. de, Bones, V. M., Souza, I. S., \& Guimarães, N. K. (2006). Rede de relações sociais e satisfação com a vida de adultos e idosos. Psicol. Am. Lat., 5, 1-15.

Schirrmacher, F. (2004, 18 de Agosto). A ditadura dos jovens. Veja, 33, pp. 11-15.

Silberman, C., Souza, C., Wilhems, F., Kipper, L., Wu, V., Diogo, C. et al. (1995). Cognitive deficit and depressive symptoms in a community group of elderly people: A preliminary study. Revista de Saúde Pública, 29(6), 444-450.

Soares, L. (2004, 15 de Setembro). Viver mais e melhor. Veja, 37, pp. 96-108.

Veras, R. (2003). Em busca de uma assistência adequada à saúde do idoso: Revisão da literatura e aplicação de um instrumento de detecção precoce e de previsibilidade de agravos. Cad. Saúde Pública, 19(3), 705-715.

Vila, E. M. (2005). Treinamento de habilidades sociais em grupo com professores de crianças com dificuldades de aprendizagem: Uma análise sobre procedimentos e efeitos da intervenção. Dissertação de Mestrado, Programa de Pós-Graduação em Educação Especial, Universidade Federal de São Carlos, São Carlos.

Vila, E. M., Gongora, M. A. N., \& Silveira, J. M. (2003). Ensinando repertório alternativo para clientes que apresentam padrões comportamentais passivo e hostil. In C. G. de Almeida (Org.), Intervenções em grupos: Estratégias psicológicas para a melhoria da qualidade de vida (pp. 59-81). Campinas: Papirus.

Xavier, F., Ferraz, M. P. T., Bisol, L. W., Fernandes, D. D., Schwanke, C., \& Moriguchi, E. H. (2000). Octogenários de Veranópolis: As condições psicológicas, sociais e de saúde geral de um grupo representativo de idosos com mais de 80 anos residentes na comunidade. Revista AMRIGS, 44(1,2), 25-29.

Zimerman, G. I. (2000). Velhice: Aspectos biopsicossociais. Porto Alegre: Artes Médicas.

Psicol. Argum., Curitiba, v. 32, n. 76, 23-31 